\title{
Phylogeny of Flabellulidae (Amoebozoa: Leptomyxida) inferred from SSU rDNA sequences of the type strain of Flabellula citata Schaeffer, 1926 and newly isolated strains of marine amoebae
}

\author{
Iva Dyková ${ }^{1,2}$, Ivan Fiala $^{1,2}$, Hana Pecková $^{1}$ and Helena Dvořáková ${ }^{1}$ \\ ${ }^{1}$ Institute of Parasitology, Biology Centre of the Academy of Sciences of the Czech Republic, Branišovská 31, 37005 České \\ Budějovice, Czech Republic; \\ ${ }^{2}$ Faculty of Science, University of South Bohemia, Branišovská 31, 37005 České Budějovice, Czech Republic
}

Key words: Amoebozoa, Leptomyxida, Flabellulidae, Flabellula citata, SSU rDNA phylogeny

\begin{abstract}
New strains of non-vannellid flattened amoebae isolated from fish, an invertebrate and the marine environment were studied together with Flabellula citata Schaeffer, 1926 selected by morphology as a reference strain. The study revealed a paucity of features distinguishing individual strains at the generic level, but clearly evidenced mutual phylogenetic relationships within the assemblage of strains as well as their affiliation to the Leptomyxida. In this study, the SSU rDNA dataset of leptomyxids was expanded and a new branching pattern was presented within this lineage of Amoebozoa. Sequences of three newly introduced strains clustered in close relationship with the type strain of F. citata, the type species of the genus. Three strains, including one resembling Flamella sp., were positioned within a sister-group containing Paraflabellula spp. Results of phylogenetic analysis confirmed doubts of previous authors regarding generic assignment of several Rhizamoeba and Ripidomyxa strains.
\end{abstract}

Naked amoebae with fan-shaped trophozoites flattened to a substrate were described in the early period of amoeba research. At that time, Schaeffer (1926) established the genus Flabellula for marine amoebae with triangular or flabelliform trophozoites.

Amendments to the generic diagnosis of Flabellula Schaeffer, 1926 by Bovee (1965) made it consistent with the diagnosis of the type species ( $F$. citata Schaeffer, 1926) and lead to the erection of the genus Vannella Bovee, 1965. Bovee (1970) also removed Flabellula from the Mayorellidae and established the family Flabellulidae. The re-diagnosis of the genus Flabellula that included also ultrastructural features was published by Page in 1980. Page (1983) later unified three genera, Flabellula, Paraflabellula Page et Willumsen, 1983, and Flamella Schaeffer, 1926, within the family Flabellulidae Bovee, 1970. In addition, Flabellula citata Schaeffer, 1926, Paraflabellula reniformis (Schmoller, 1964) and Flamella magnifica Schaeffer, 1926 were designated as the type species of the respective genera. The inclusion of Flamella Schaeffer, 1926 in the Flabellulidae was confirmed by Michel and Smirnov (1999), who amended the generic diagnosis and described two new species (F. aegyptia and F. lacustris). The five species of Flabellula were used by Smirnov and Goodkov (1999) to exemplify "flabellate" morphotype of the Gymnamoebia, whereas for species of the genera Paraflabellula and Flamella they defined "paraflabellulian" morphotype.
In phylogenetic analyses based on SSU rDNA sequences, two representatives of Flabellulidae, P. reniformis and P. hoguae (Sawyer, 1975) were presented as members of Leptomyxa-Hartmannella (LH) clade in Amaral Zettler et al. (2000). The same phylogenetic position of these two sequences was recognized by Bolivar et al. (2001) when they investigated the ancestry of Gymnamoebae. The LH lineage persisted in later analysis of the Gymnamoebia (Peglar et al. 2003) but contained only the sequence of $P$. hoguae. A clade denominated "Leptomyxoidea" with sequences of two Paraflabellula spp., Rhizamoeba sp. and Leptomyxa reticulata Goodey, 1914 was presented by Cavalier-Smith et al. (2004) in their revision of the higher-level classification of Amoebozoa. These authors identified this clade within the superfamily Leptomyxoidea Pussard et Pons, 1976 and divided it into Flabellulidae Bovee, 1970 and Leptomyxidae Pussard et Pons, 1976. In the high-rank phylogenetic classification of Amoebozoa, Smirnov et al. (2005) mentioned the genus Flabellula as "probably belonging" to the order Leptomyxida. This was due to the lack of representation of Flabellula gene sequences but evident morphological similarities of Flabellula with other genera assigned to the families Leptomyxidae Pussard et Pons, 1976 and Flabellulidae Bovee, 1970. In the sequence analysis of 27 strains of Lobosea, Smirnov et al. (2008) introduced CCAP1570/42 strain as a new species of Leptomyxida, most accurately representing Rhizamoeba saxonica Page, 1974. In the same

Address for correspondence: I. Dyková, Institute of Parasitology, Biology Centre of the Academy of Sciences of the Czech Republic, Branišovská 31, 37005 České Budějovice, Czech Republic. Phone: +420 387775 423; Fax: +420 385 310 388; E-mail: iva@paru.cas.cz 
paper, Smirnov et al. (2008) questioned the generic assignment of the ATCC 50742 strain to Rhizamoeba, the validity of the Ripidomyxa sp., and excluded Flamella from Leptomyxida.

Although the search for correlation between phylogenetic and morphological groupings of amoeboid protists has motivated the research on these organisms for more than a decade, gene sequences of the strain representatives of many genera have not been included in phylogenetic tree reconstructions. Some clades are poorly represented, and the gene sequences of some species that are morphologically assigned to the same genus are in distant phylogenetic positions and await revision of their generic identification.

This study was undertaken with the aim to incorporate into phylogenetic analyses the SSU rDNA sequence of the type strain of Flabellula citata Schaefer, 1926 and clarify phylogenetic positions of other strains with similar light microscopical and ultrastructural features.

\section{MATERIALS AND METHODS}

The study is based on six strains of marine amoebae we isolated from the tissues of three species of fishes, turbot, Psetta maxima (L.), (Pleuronectiformes: Scophthalmidae); triggerfishes, Sufflamen verres (Gilbert et Starks) and Balistes polylepis Steindachner (both Tetraodontiformes: Balistidae); starfish, Porania pulvillus (O. F. Müller) (Asteroida: Poranidae); and from sediments and net material of sea floating cages used in Atlantic salmon aquaculture (Table 1). These strains were selected by their light microscopical features, which were similar to those of flabellulids. In addition, the type strain of Flabellula citata (CCAP 1529/2), the type species of the genus, obtained from UK National Culture Collection (UKNCC), was included in the study. New strains were isolated using MY75S (Malt \& Yeast Extract-75\% seawater) agar (Catalogue of the UKNCC, 2001) with the content of extracts reduced to half. Primary isolates of amoebae were either directly transferred onto MY75S agar or after the amount of contaminating bacteria was reduced using MY75S agar with reduced content of extracts. Subculturing of strains and clonal cultures derived from them were accomplished on MY75S agar that was not seeded with bacteria during the whole period of subculturing. The same method of subculturing was also used for F. citata (CCAP 1529/2), F. trinovantica (CCAP 1529/4) and F. demetica (CCAP 1529/3) strains. The cultures of the latter two strains (purchased also from
UKNCC) were heavily contaminated with bacteria and they failed to propagate, therefore only their morphology could be observed.

Light microscopical observations of living trophozoites were made in hanging drop preparations, using the Olympus Nomarski differential interference contrast (DIC). Hoechst 33258 (Sigma) fluorescent dye was used to visualise nuclei in trophozoites after their fixation in $95 \%$ ethanol. The images of trophozoites characteristic of individual strains were selected from sets counting 40 to 60 trophozoites for each strain. Samples for transmission electron microscopy were prepared as previously described (Dyková et al. 2003). Ultrathin sections were examined with a JEOL JEM 1010 electron microscope operating at $80 \mathrm{kV}$. Images were collected with MEGAview II soft imaging system using analySIS ${ }^{\circledR}$ software. Typical ultrastructural features were studied in ultrathin sections from trophozoites fixed in two to four different periods of subculturing.

DNA was extracted from pelleted trophozoites using the DNeasy ${ }^{\mathrm{TM}}$ Tissue Kit (Qiagen GmbH, Germany) according to the manufacturer's protocol. Universal eukaryotic primers (5' ACCTGGTTGATCCTGCCAG 3' and 5' CTTCCGCAGGTTCACCTACGG 3') (Barta et al. 1997) were used for amplification of the SSU rRNA gene. PCR was carried out in $25 \mu \mathrm{l}$ reaction volume using $10 \mathrm{pmol}$ of each primer, $250 \mu \mathrm{M}$ of each dNTP, $2.5 \mu 110 \times$ PCR Buffer (Top-Bio, Czech Republic) and 1 Unit of TaqDNA polymerase (Top-Bio, Czech Republic). The reactions were run on a Tpersonal Thermocycler (Biometra). The thermal cycling conditions consisted of initial denaturation at $95^{\circ} \mathrm{C}(5 \mathrm{~min}), 30$ cycles of denaturation at $94^{\circ} \mathrm{C}(1 \mathrm{~min})$, annealing at $48^{\circ} \mathrm{C}(1.5 \mathrm{~min})$ and extension at $72^{\circ} \mathrm{C}(2 \mathrm{~min})$ followed by a final extension at $72^{\circ} \mathrm{C}(10 \mathrm{~min})$. Following visualisation of PCR products via gel electrophoresis, amplification products were extracted from the agarose using JETQUICK Gel Extraction Spin Kit (Genomed, Germany), then cloned into $\mathrm{pCR}^{\circledR} 2.1$ TOPO Cloning vector using the TOPO-TA Cloning Kit (Invitrogen) and sequenced on an automatic sequencer CEQ ${ }^{\mathrm{TM}} 2000$ using CEQ DTCS Dye Kit (Beckman Coulter) according to the manufacturer's protocol. The complete SSU rDNA sequence was obtained stepwise using a combination of flanking and internal primers as mentioned elsewhere (Dyková et al., in press).

SSU rDNA sequences were aligned by Clustal_X program (Thompson et al. 1997) using gap opening/gap extension penalties $8 / 2$. Total number of characters in the dataset was 1832 (122 ambiguous positions were removed). The phylogenetic trees were constructed by maximum parsimony (MP) and maximum likelihood (ML) methods using PAUP*, Ver-

Table 1. Strains characterised in the study and their origin.

\begin{tabular}{llll}
\hline Strain/clone & Origin / Host origin & Local origin & Isolated/cloned \\
\hline SEDF1/I & Sediments & Tasmania, Salmo salar farm & 07.04.02/May 2003 \\
STAR2 & Stomach / Porania pulvillus & Norway, Vevang & 03.10.05/Nov. 2005 \\
NETC3/I & Net material of floating cages & Tasmania, Salmo salar farm & 17.02.04/Apr. 2004 \\
M4M/I & Gill tissue / Sufflamen verres & Mexico, Mazatlan & 03.07.00/Sept. 2004 \\
M9M/I & Gill tissue / Balistes polylepis & Mexico, Mazatlan & 03.07.00/Oct. 2004 \\
SMA17/I & Gill tissue / Psetta maxima & NW Spain, P. maxima farm & 25.08.00/Mar. 2003 \\
CCAP1529/2 & Marine tidal pool & USA, Maine (isolator F.C. Page) & 1969 \\
\hline
\end{tabular}




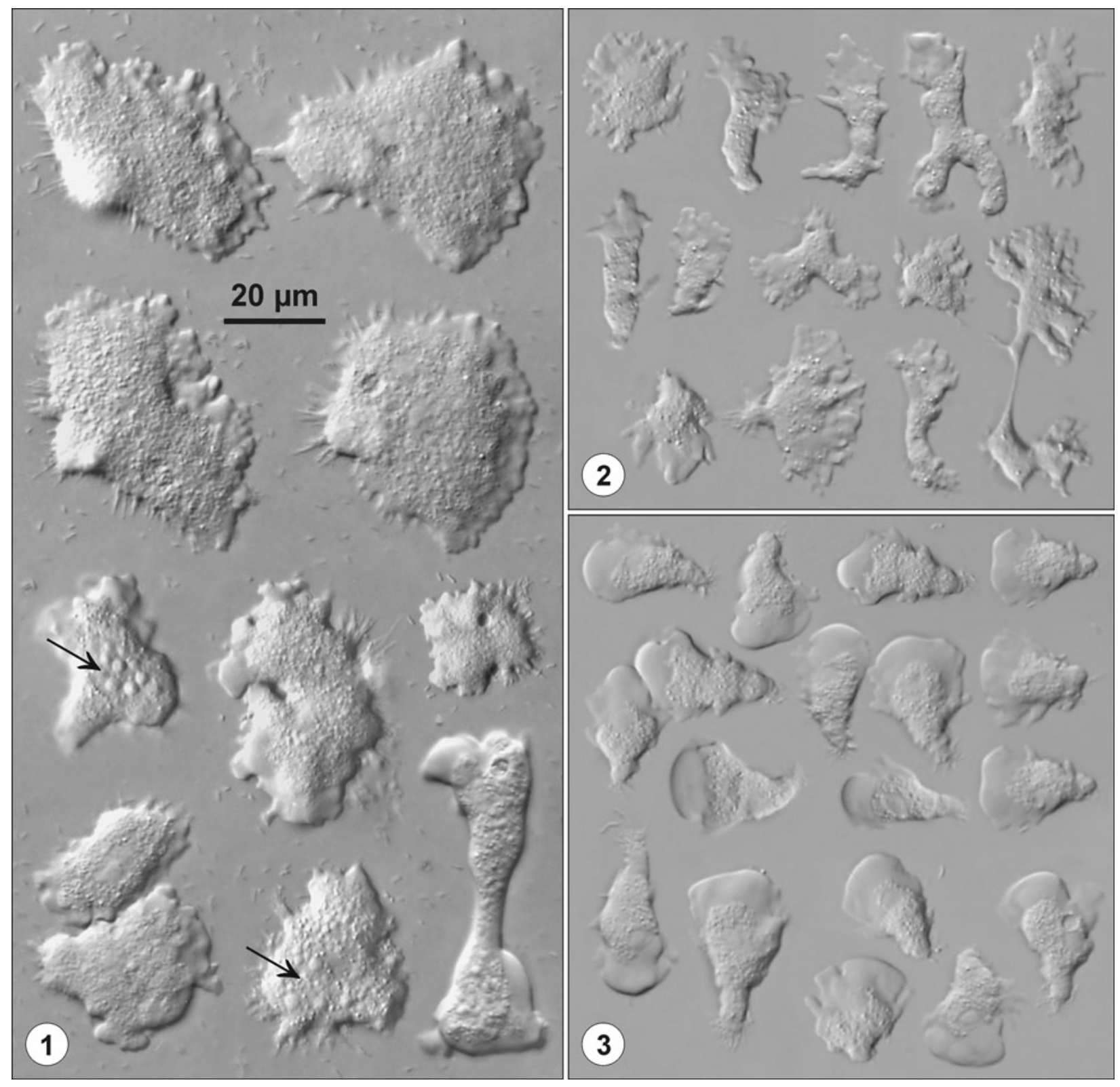

Figs. 1-3. Trophozoites of one-week-old cultures of flabellulid amoebae under study as observed in hanging drop preparations. Fig. 1. Type strain of Flabellula citata Schaeffer, 1926 from UK National Culture Collection (CCAP 1529/2). Multinucleate trophozoites are marked with arrows. Fig. 2. M9M strain. Fig. 3. M4M strain. Figs. 1-3: same scale.

sion $4.0 \mathrm{~b} 10$ (Swofford 2001). Both MP and ML were per formed with heuristic search with tree bisection-reconnection branch swapping and random addition of taxa (10 replications). Gaps were treated as missing data. For MP the matrix was analysed under transition/transversion ratio $=1: 2$. ML analysis was performed with the best fitting model of evolution $(\mathrm{GTR}+\Gamma+\mathrm{I})$ and parameters computed by the likelihood ratio test implemented in the Modeltest v. 3.06 (Posada and Crandal 1998). Clade support was assessed with bootstrapping of 1000 replicates for MP and 500 replicates for ML.

\section{RESULTS}

\section{Light microscopy and ultrastructure}

Trophic cells of Flabellula citata (CCAP strain 1529/2) had the size consistent with the range given for three strains of this species by Page (1971) and revealed all morphological features described by Page (1971, 1980). In contrast to the living locomotive forms documented by Page $(1971,1980)$, trophozoites of F. citata 


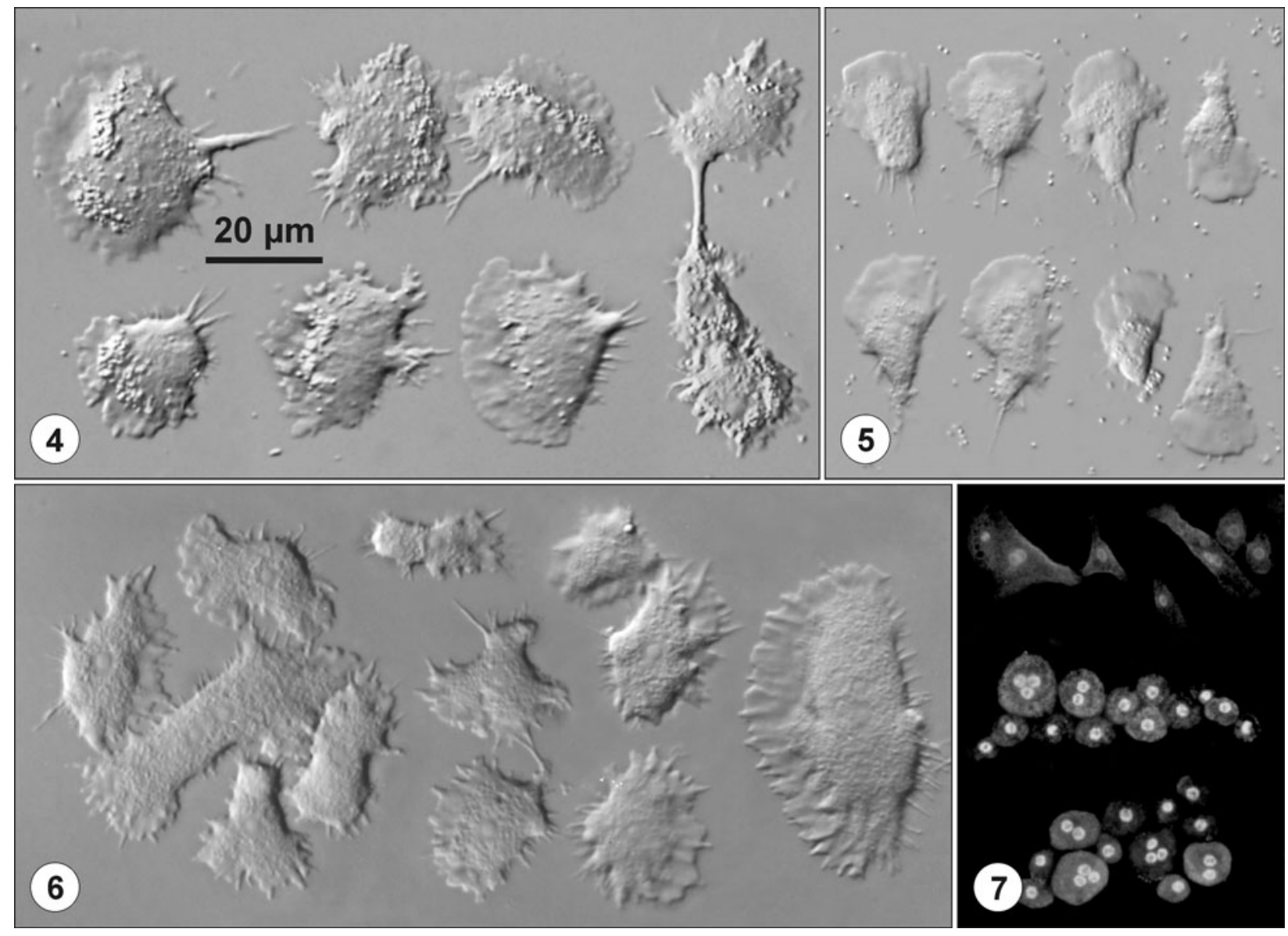

Figs. 4-6. Trophozoites of one-week-old cultures of flabellulid amoebae under study as observed in hanging drop preparations. Fig. 4. NETC3 strain. Fig. 5. STAR2 strain. Fig. 6. SEDF strain. Fig. 7. Trophozoites of SEDF strain with nuclei stained with Hoechst 33258 fluorescent dye. Figs. 4-6: same scale; Fig. 7: not to scale.

subcultured in our laboratory, showed an irregular hyaloplasmic zone slightly narrowed in favour of granuloplasm, and uroid filaments finer than documented for this species in previous papers (Fig. 1). The former difference was most probably due to a heavy load of bacteria in the cytoplasm, the latter was due to different methods of observation and documentation (bright field used by Page versus Nomarski DIC used in our study). The shape of flabelliform trophozoites varied to the same degree as documented by Page (1971), from closed to open fan-like forms. In 5-day-old cultures, the majority of trophozoites contained more than one nucleus; in some cases up to six nuclei were observed in one trophozoite (Figs. 1, 6, 7).

The other strains included in the study revealed morphological features of the family Flabellulidae Bovee, 1970 as described by Page (1980, 1983). When compared with the CCAP strain of F. citata, declared as the type strain of the type species (Page 1971, 1980), trophozoites of new strains differed in having either a more indented outline of hyaloplasmic cell periphery (strain M9M in Fig. 2 or SEDF in Fig. 6) or an almost smooth outline (M4M and STAR2) (Figs. 3 and 5, respectively).
The anterior hyaline zone of locomotive forms was mostly well developed. It represented nearly half the total length of trophozoites in M4M and STAR2 strains. Uroid filaments differed in their thickness and length, being almost invisible in M4M strain, while trophozoites of NETC3 strain sometimes possessed a slightly elongated posterior region with fine uroid filaments (Fig. 4). More than one nucleus was observed in numerous trophozoites, using Nomarski DIC and fluorescent dye (Figs. 1, 7). Multinucleate trophozoites were observed frequently in F. citata (Fig. 1). Trophozoites of all strains included in the study were sensitive to the intense light used for Nomarski DIC and transformed very fast into irregularly spherical forms. Floating forms with short pseudopodia (if present) did not last long. Cyst formation was never observed in our set of strains. At lower magnifications, cysts could be confused with spherical dying stages.

Transmission electron microscopy revealed that the strains under study share most of ultrastructural features. The cell membrane was covered with a very thin surface coat (Fig. 9). The cytoplasm, rich in agranular endoplasmic reticulum, also contained ribosomes that 

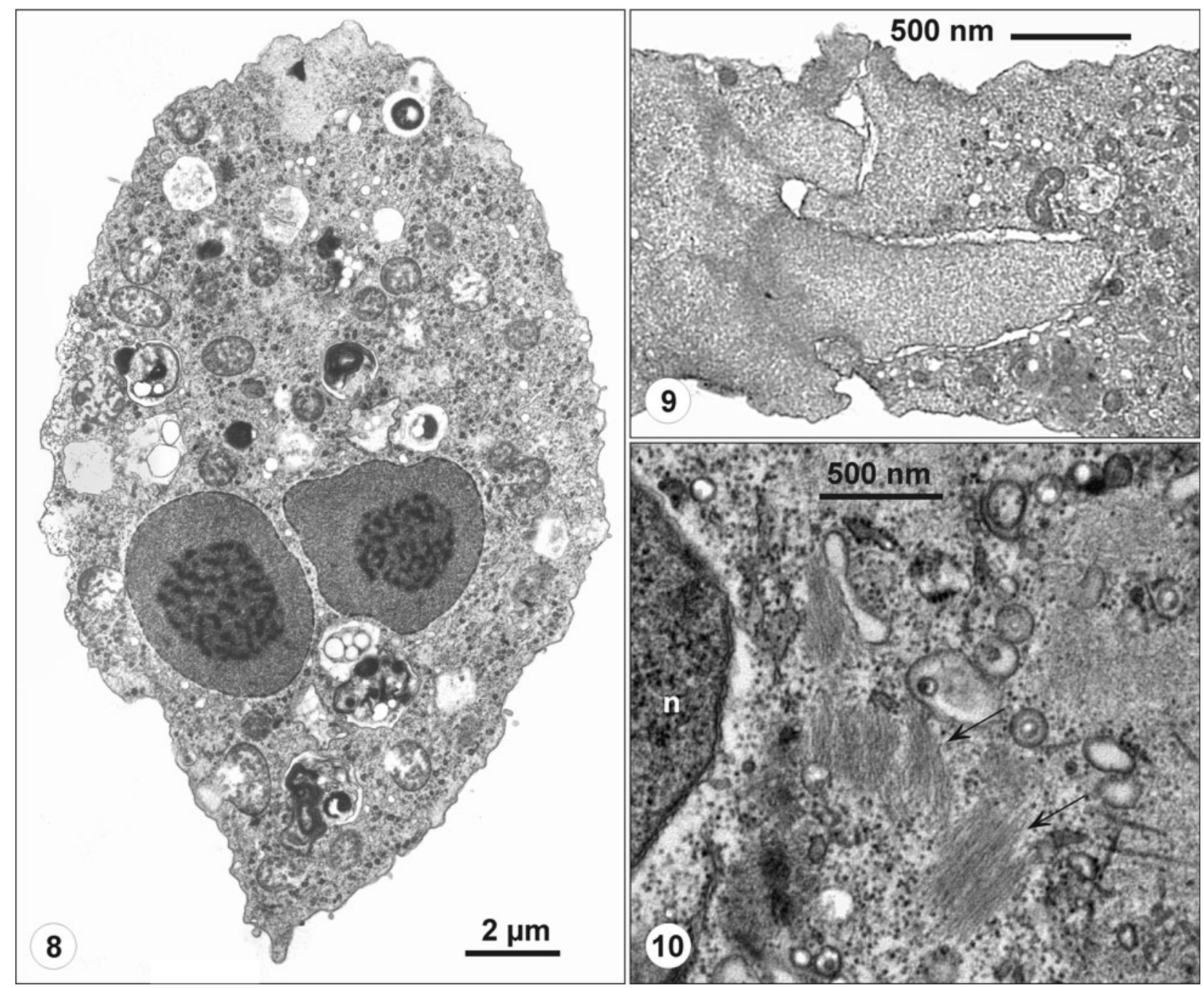

Figs. 8-10. Ultrastructure of flabellulid amoebae under study. Fig. 8. Binucleate trophozoite of SEDF strain. Fig. 9. Close apposition of trophozoites of Flabellula citata strain CCAP 1529/2, suggesting their fusion. Fig. 10. Cytoplasm of Flabellula citata trophozoite with longitudinally oriented fibrilar structures (arrows) in the vicinity of nucleus (n).

were closely associated with the membranes of endoplasmic reticulum (Fig. 15) or freely accumulated in the cytoplasm. The cytoplasm also contained numerous, mostly rounded or ovoid mitochondria with tubular branching cristae, multiple vacuoles with remnants of food, bacteria, and microbodies of unknown origin (Figs. 8, 16). The parallelly oriented microfibrils were observed in the cytoplasm of the type strain of $F$. citata (Fig. 10). The single rounded nucleus that contained centrally located well-condensed nucleolar material was found in ultrathin sections rather exceptionally (Fig. 11). Elongate and lobed nuclei with dense nucleolar material divided in two or more distantly located portions were more frequent (Figs. 12-14, 17). Binucleate trophozoites predominated in one-week-old subcultures but also multinucleate trophozoites were observed using electron microscopy (Fig. 8).

Based on light microscopical features, the six strains included in the study in addition to the type strain of
F. citata could be safely assigned to the family Flabellulidae. Although repeated observations of trophozoites (from different passages of each strain) revealed that the interstrain shape differences were more pronounced than those seen within one strain, safe generic diagnosis based on morphology alone was impossible. Tentatively, the STAR2 and NETC3 strains could be assigned to Paraflabellula. Based on comparison that included also trophozoites of $F$. trinovantica Page, 1980 and F. demetica Page, 1980 (UKNCC strains), the M9M, M4M and SMA17/I strains could be assigned to Flabellula, and trophozoites of SEDF strain resembled those of the genus Flamella.

Due to the lack of features clearly discriminating the genera Flabellula, Paraflabellula and Flamella at the light microscopical and ultrastructural levels and due to the fact that also documentation in previous papers was not always congruent with descriptions of flabellulids, generic appurtenance of the newly isolated strains of 


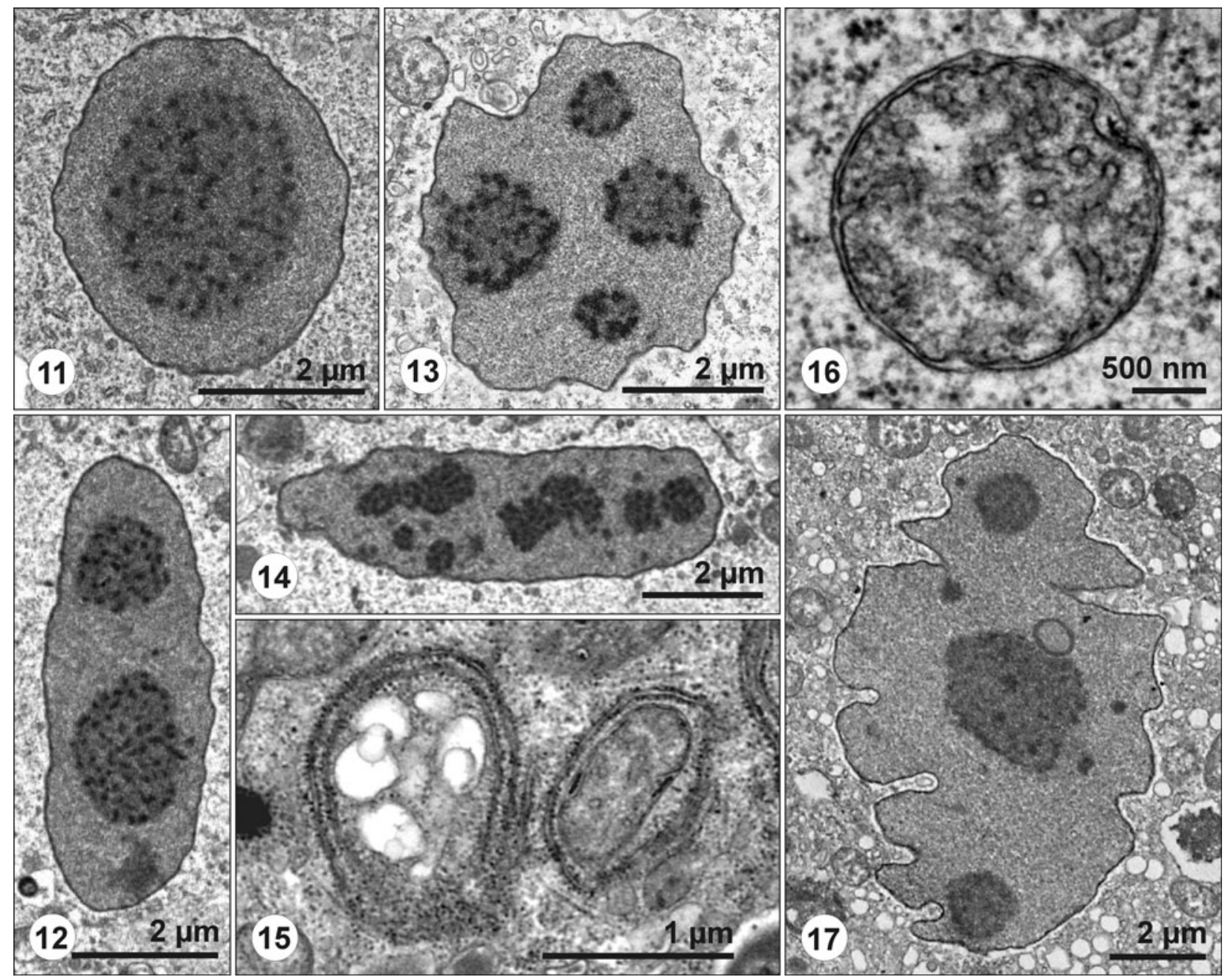

Figs. 11-17. Ultrastructure of flabellulid amoebae under study. Fig. 11. Rounded nucleus with centrally located nucleolar material (trophozoite of M9M strain). Fig. 12. Elongated nucleus with nucleolar material divided into two separated portions (M9M strain). Fig. 13. Nucleus of irregular shape with nucleolar material divided in four parts (M4M strain). Fig. 14. Elongate nucleus with dispersed nucleolar material (M9M strain). Fig. 15. Cisternae of endoplasmic reticulum surrounding vesicular part of cytoplasm (left) and mitochondrion (right) (STAR2 strain). Fig. 16. Mitochondrion with tubular branching cristae characteristic of flabellulid amoebae (M9M strain). Fig. 17. Lobed nucleus with dispersed nucleolar material in the trophozoite of Flabellula citata (CCAP1529/2 strain).

flabellulids remained opened for future research, when more data (probably molecular markers) are accumulated.

\section{SSU rDNA sequences and phylogeny}

Sequence data obtained in this study were deposited in the GenBank under accession numbers EU852652EU852658. Length of sequences ranged from a minimum of $1866 \mathrm{bp}$ in STAR2 strain, to a maximum of 1876 bp in SMA17/I strain. GC content ranged from a minimum of $40.8 \%$ to a maximum of $45.5 \%$ in NETC3/I and M9M/I, respectively. The sequence similarity calculated for strains under study is rather high (Table 2).
Table 2. The sequence similarity (\%) within strains under study.

\begin{tabular}{llccccccc}
\hline & \multicolumn{1}{c}{1} & 2 & 3 & 4 & 5 & 6 & 7 \\
\hline 1 & CCAP1529/2 & - & & & & & & \\
2 & SMA17/I & 97.82 & - & & & & \\
3 & M4M/I & 98.13 & 99.38 & - & & & \\
4 & M9M/I & 98.13 & 99.38 & 99.58 & - & & \\
5 & STAR2 & 93.32 & 93.39 & 93.01 & 93.01 & - & & \\
6 & NETC3/I & 93.38 & 93.50 & 93.23 & 93.13 & 98.93 & - & \\
7 & SEDF/I & 93.55 & 93.57 & 93.19 & 93.19 & 98.21 & 98.40 & - \\
\hline
\end{tabular}




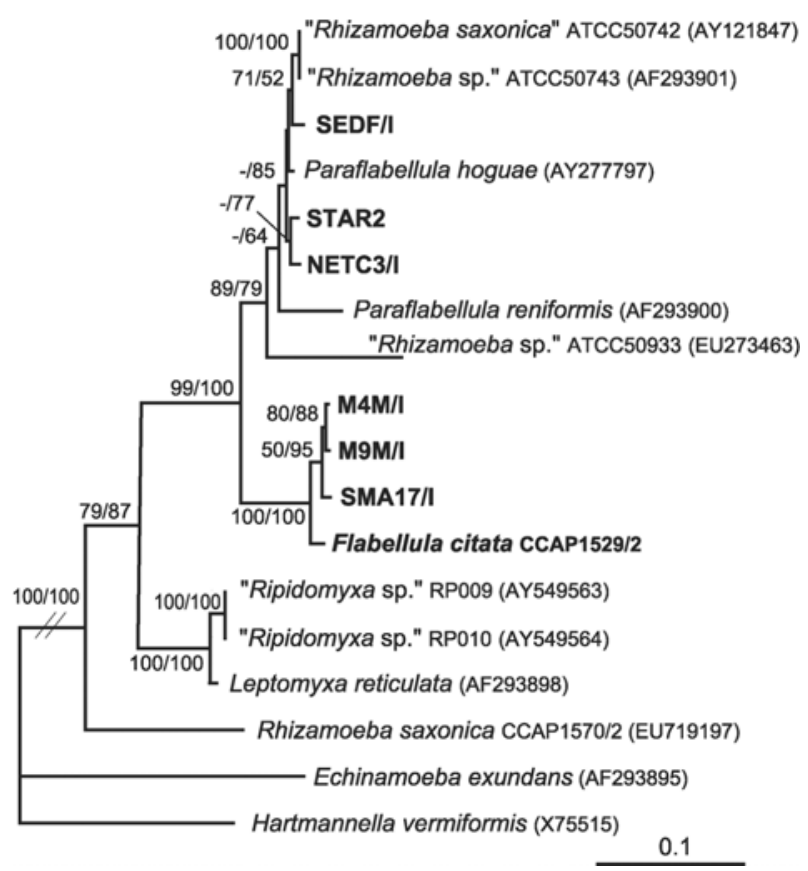

Fig. 18. The maximum likelihood tree $(\mathrm{GTR}+\Gamma+\mathrm{I}$ model, $-\ln =7659.291, \alpha$ shape parameter $=0.62$, proportion of invariable sites $=0.40$ ) of the SSU rDNA sequences of leptomyxid amoebae with Echinamoeba exundans and Hartmannella vermiformis as outgroup. Bootstrap values (ML and MP $\mathrm{Ts} / \mathrm{Tv}=1: 2)$ are indicated for the nodes gaining more than $50 \%$ support. The branch leading from outgroup to leptomyxids is three times shortened. GenBank accession numbers are in parentheses. The scale bar is given under the tree. Taxa whose names are given within quotation marks are suspected not to have been correctly determined.

The results of phylogenetic analyses are shown in Fig. 18. This reconstructed tree provided the first molecular evidence for the position of the F. citata type strain (CCAP 1529/2) and closely related strains SMA17/I, M9M/I and M4M/I within leptomyxids. The sequences of other newly isolated strains fell into a sister clade together with sequences of Paraflabellula strains that traditionally represent leptomyxid line in amoebozoan phylogenetic reconstructions. The division of strains under study into two sister clades is evident, nevertheless, composition of Paraflabellula clade indicates possible future changes in the branching pattern (see in Discussion).

\section{DISCUSSION}

As information on phylogenetic relationships within Amoebozoa continues to accumulate, the gaps in our knowledge become more visible and stimulate the effort directed to amoebae thus far neglected. The selection of non-vannellid flattened amoebae from our collection of strains was not difficult. In some strains it was possible to recognise features discriminating trophozoites from vannellid type even at low magnification while check- ing agar plate cultures. Difficulties occurred in attempting to make a generic diagnosis of selected strains based on light microscopical and ultrastructural features, despite of the rather exceptional opportunity to compare our strains with the type strain, Flabellula citata. Controversies that emerged from data in the literature and comparison of live trophozoites enabled only the tentative generic assignment of marine amoebae under study. Noticeable similarity of the type species of Flabellula and Paraflabellula was stressed in description of Paraflabellula reniformis (Page and Willumsen 1983). Fibrils oriented in the cytoplasm in dense parallel bunches were taken as the most distinctive feature of $P$. reniformis by Page and Willumsen (1983). In this study, they were found in trophozoites of F. citata. Nonfurcate subpseudopodia produced from broad hyaloplasmic lobopodium (included in the original description of $P$. reniformis) were expected by the latter authors in other unrelated groups of amoebae. When images of the trophozoites characteristic of flabellulid species were compared, Flabellula baltica Smirnov, 1999 was found to be documented by trophozoite with prolonged posterior part, almost identical with Paraflabellula type (Smirnov 2007). In "An illustrated list of basic morphotypes" (Smirnov and Goodkov 1999), trophozoite of $F$. baltica resembles those of $F$. citata. Among trophozoites characteristic of the SEDF strain (Fig. 6) were found individuals identical with Schmoller's (1964) image of Rugipes reniformis, with one trophozoite of $P$. reniformis presented by Page (1983) and a certain similarity was noticed also with the line drawing of Hyalodiscus actinophorus (currently Cochliopodium actinophorum) by Page (1968). We think that trophozoites of SEDF strain are most similar to those of Flamella described by Michel and Smirnov (1999). Unfortunately, SSU rDNA sequences of similar, morphologically described strains are missing. It only can be noted that Smirnov et al. (2008) excluded Flamella from Leptomyxida due to the lack of monopodial locomotive form declared by Michel and Smirnov (1999) as absent in Flamella spp. This fact did not fit the concept of Tubulinea (formerly Lobosea) presented by Smirnov et al. (2005). In the context of the family name Flabellulidae, the replacement of the class name Discosea (Cavalier-Smith et al. 2004) with Flabellinea that does not accommodate the Flabellulidae (Smirnov et al. 2008) does not seem ideal.

Multinucleate trophozoites observed in flabellulid strains under study were described also by Page (1971), who found a big proportion of binucleate trophozoites $(12 \%)$ in Flabellula calkinsi. Less frequent occurrence of binucleate trophozoites was mentioned in the description of P. reniformis (Page and Willumsen 1983). Michel and Smirnov (1999) observed spontaneous fusions of cells resulting in formation of locomotive plasmodium in Flamella lacustris, while in culture of Flamella aegyptia they observed only fragmentation of multinu- 
cleate stages. Cell fusion and subsequent parting was observed also in Flabellula baltica (Smirnov and Goodkov 1999). The cell fusion observed in Leptomyxa reticulata was classified by Seravin and Goodkov (1984) as pseudocopulation. Pseudocopulation together with plasmodization and pseudoconjugation were presented by the latter authors as the principal types of agamic cell fusion that occurs among protozoa. The origin of multinucleate trophozoites of flabellulids observed also in this study undoubtedly deserves further detailed study.

Phylogenetic analysis that clearly evidenced affiliation of the Flabellula citata type strain (CCAP 1529/2) and of closely related strains with leptomyxids, showed also some confusing data. Gene sequences of three newly introduced strains branched within the clade that in addition to two Paraflabellula strains contains sequences of organisms that, most probably, were incorrectly determined. Since recently re-examined CCAP
$1570 / 2$ strain has been denoted as the only valid representative of Rhizamoeba saxonica (Smirnov et al. 2008), the other ATCC "Rhizamoeba" strains, branching in distant positions, should be re-examined and properly determined. The same applies to Ripidomyxa strains that are closely related to Leptomyxa reticulata. This fact has already been mentioned by Hewett (2006).

Although this study has given some convincing data, the apparent similarity of genera described within the family Flabellulidae is far from being analysed in detail. More data are needed to make a revision of congruence between morphological and molecular characteristics.

Acknowledgements. The Ministry of Education, Youth and Sports (MSM 6007665801) and Research projects of the Institute of Parasitology, Academy of Sciences of the Czech Republic (Z60220518 and LC522) funded this research.

\section{REFERENCES}

Amaral Zettler L.A., Nerad T.A., O'Kelly C.J., Peglar M.T., Gillevet P.M., SiLBERMAN J.D., Sogin M.L. 2000: A molecular reassessment of the leptomyxid amoebae. Protist 151: 275-282.

Barta J.R., Martin D.S., Liberator P.A., DAShKewitz M., Anderson J.W., Deighner S.D., Elbrecht A., PerkinsBARROW A., JENKINS M.C., DANFORTH H.D., RUFF M.D., PROFOUS-JUCHELKA H. 1997: Phylogenetic relationships among eight Eimeria species infecting domestic fowl inferred using complete small subunit ribosomal DNA sequences. J. Parasitol. 83: 262-271.

BOlivar I., FAHRNi J.F., SMIRNOV A.V., PAWLOWSKI J. 2001: SSU rRNA-based phylogenetic position of the genera Amoe$b a$ and Chaos (Lobosea, Gymnamoebida): the origin of Gymnamoebae revisited. Mol. Biol. Evol. 18: 2306-2314.

BOVEE E.C. 1965: An emendation of the amoeba genus Flabellula and description of Vannella gen. nov. Trans. Am. Microsc. Soc. 84: 217-221.

BOVEE E.C. 1970: The lobose amoebas. I. A key to the suborder Conopodina Bovee and Jahn, 1966 and descriptions of thirteen new or little known Mayorella species. Arch. Protistenkd. 112: 178-227.

CAvalier-Smith T., ChaO E.E., OAtes B. 2004: Molecular phylogeny of Amoebozoa and the evolutionary significance of the unikont Phalansterium. Eur. J. Protistol. 40: 21-48.

DYKOVÁ I., FIALA I., LOM J., LUKEŠ J. 2003: Perkinsiela amoebae-like endosymbionts of Neoparamoeba spp., relatives of the kinetoplastid Ichthyobodo. Eur. J. Protistol. 39: 37-52.

DYKOVÁ I., PECKOVÁ H., KOSTKA M.: Introduction of Mayorella gemmifera Schaeffer, 1926 into phylogenetic studies of Amoebozoa. Acta Protozool. (In press.)

HewetT M.K. 2006: Characterisation of bacterial symbionts in amoebae. A thesis submitted for the degree of Doctor of Philosophy. School of Pharmaceutical, Molecular and Biomedical Sciences, University of South Australia and CRC for Water Quality and Treatment, $96 \mathrm{pp}$.

Michel R., SMIRNOV A.V. 1999: The genus Flamella Schaeffer, 1926 (Lobosea, Gymnamoebia), with description of two new species. Eur. J. Protistol. 35: 400-410.
PAGE F.C. 1968: Generic criteria for Flabellula, Rugipes and Hyalodiscus, with descriptions of species. J. Protozool. 15: 9 26.

PAGE F.C. 1971: Two marine species of Flabellula (Amoebida, Mayorellidae). J. Protozool. 18: 37-44.

PAGE F.C. 1980: A light- and electron microscopical comparison of limax and flabellate marine amoebae belonging to four genera. Protistologica 16: 57-78.

PAGE F.C. 1983: Marine Gymnamoebae. Institute of Terrestrial Ecology, Culture Centre of Algae and Protozoa. Cambridge, England, $54 \mathrm{pp}$.

Page F.C., Willumsen N.B.S. 1983: A light- and electronmicroscopical study of Paraflabellula reniformis (Schmoller, 1964), type species of a genus of amoebae (Amoebida, Flabellulidae) with subpseudopodia. Protistologica 19: 567-575.

Peglar M.T., Amaral ZeTtLer L.A., ANDERSON O.R., Nerad T.A., Gillevet P.M., Mullen T.E., Frasca JR. S., SilberMAN J.D., O'KeLLY C.J., SOGIN M.L. 2003: Two new smallsubunit ribosomal RNA gene lineages within the subclass Gymnamoebia. J. Eukaryot. Microbiol. 50: 224-232.

PosadA D., CRANDAll K.A. 1998: Modeltest: testing the model of DNA substitution. Bioinformatics 14: 817-818.

SAWYER T.K. 1975: Marine amoebae from surface waters of Chincoteague Bay, Virginia: two new genera and nine species within the families Mayorellidae, Flabellulidae and Stereomyxidae. Trans. Am. Microsc. Soc. 94: 71-92.

SCHAEFFER A.A. 1926: Taxonomy of the Amebas. Publ. No. 345, Dept. Mar. Biol., Carnegie Institution of Washington 24: 3112.

SCHMOLLER H. 1964: Beschreibung einiger Kulturamöben mariner Herkunft. J. Protozool. 11: 497-502.

SERAVIN L.N., GoODKOV A.V. 1984: The main types and forms of agamic cell fusion in protozoa. Tsitologia 26: 123-131. (In Russian.)

SMIRNOV A.V. 2007: Cryptic freshwater amoeba species in the bottom sediments of Nivå Bay (Oresund, Baltic Sea). Eur. J. Protistol. 43: 87-94.

SMIRNOV A.V., GOODKOV A.V. 1999: An illustrated list of basic morphotypes of Gymnamoebia (Rhizopoda, Lobosea). Protistology 1: 20-29. 
SMIRNOV A., NASSONOVA E., BERNEY C., FAHRNI J., BOLIVAR I, PAWLOWSKI J. 2005: Molecular phylogeny and classification of the lobose amoebae. Protist 156: 129-142.

SMIRNOV A.V., NASSONOVA E.S., CAVALIER-SMITH T. 2008: Correct identification of species makes the amoebozoan rRNA tree congruent with morphology for the order Leptomyxida Page, 1987; with description of Acramoeba dendroida n. g., n. sp., originally misidentified as 'Gephyramoeba sp'. Eur. J. Protistol. 44: 35-44.

SWOFFORD D.L. 2001: PAUP*: Phylogenetic Analysis Using Parsimony, Version 4.0b10. Sinauer Associates, Sunderland, MA.

Received 25 June 2008
TEKLE Y.I., GRANT J., ANDERSON O.R., NERAD T.A., COLE J.C. PATTERSON D.J., KATZ L.A. 2008: Phylogenetic placement of diverse amoebae inferred from multigene analyses and assessment of clade stability within "Amoebozoa" upon removal of varying rate classes of SSU-rDNA. Mol. Phylogenet. Evol. 47: 339-352.

Thompson J.D., Gibson T.J., Plewniak F., Jeanmougin F., HIGGINS D.G. 1997: The CLUSTAL_X windows interface: flexible strategies for multiple sequence alignment aided by quality analysis tools. Nucl. Acids Res. 25: 4876-4882.

Accepted 15 August 2008 